\title{
Tuning of magnetic properties in cobalt ferrite nanocrystals
}

\author{
Y. Cedeño-Mattei, ${ }^{1, a)}$ O. Perales-Perez, ${ }^{2}$ M. S. Tomar, ${ }^{3}$ F. Roman, ${ }^{1}$ P. M. Voyles, ${ }^{4}$ \\ and W. G. Stratton ${ }^{4}$ \\ ${ }^{1}$ Department of Chemistry, University of Puerto Rico, Mayagüez, Puerto Rico 00681-9019, USA \\ ${ }^{2}$ Department of Engineering Science and Materials, University of Puerto Rico, Mayagüez, \\ Puerto Rico 00681-9044, USA \\ ${ }^{3}$ Department of Physics, University of Puerto Rico, Mayagüez, Puerto Rico 00681-9016, USA \\ ${ }^{4}$ Department of Materials Science and Engineering, University of Wisconsin, Madison, \\ Wisconsin 53706-1595, USA
}

(Presented on 7 November 2007; received 11 September 2007; accepted 22 November 2007; published online 17 March 2008)

\begin{abstract}
Cobalt ferrite $\left(\mathrm{CoFe}_{2} \mathrm{O}_{4}\right)$ possesses excellent chemical stability, good mechanical hardness, and a large positive first order crystalline anisotropy constant, making it a promising candidate for magneto-optical recording media. In addition to precise control of the composition and structure of $\mathrm{CoFe}_{2} \mathrm{O}_{4}$, its practical application will require the capability to control particle size at the nanoscale. The results of a synthesis approach in which size control is achieved by modifying the oversaturation conditions during ferrite formation in water through a modified coprecipitation approach are reported. X-ray diffraction, transmission electron microscopy (TEM) diffraction, and TEM energy-dispersive x-ray spectroscopy analyses confirmed the formation of the nanoscale cobalt ferrite. $M-H$ measurements verified the strong influence of synthesis conditions on crystal size and hence, on the magnetic properties of ferrite nanocrystals. The room-temperature coercivity values increased from 460 up to 4626 Oe under optimum synthesis conditions determined from a $2^{3}$ factorial design. () 2008 American Institute of Physics. [DOI: 10.1063/1.2838215]
\end{abstract}

\section{INTRODUCTION}

Magnetic nanoparticles have been the subject of intense research because of their potential applications in highdensity magnetic recording, magnetic fluids, ${ }^{1,2}$ etc. Among the various ferrite materials for magnetic recording applications, cobalt ferrite $\left(\mathrm{CoFe}_{2} \mathrm{O}_{4}\right)$ has been widely studied because it possesses excellent chemical stability and good mechanical hardness. In addition to the precise control on the composition and structure of $\mathrm{CoFe}_{2} \mathrm{O}_{4}$, the success of its practical application relies on the capability of controlling crystal size within the superparamagnetic and single domain limits. Since the crystal size corresponding to the superparamagnetic limit has been estimated at $5 \mathrm{~nm}$, any effort to increase the coercivity in single crystals should consider the crystal growth above this critical value. ${ }^{3}$ It is known that the crystal size is related to the relative interdependence between the nucleation and growth steps, which, in turn, can strongly be affected by the solution chemistry and precipitation conditions. ${ }^{4-6}$ Several synthesis methods have been employed to control particle size and shape, ion distribution, structure and hence, the magnetic properties of ferrites. ${ }^{7-10}$ Related literature was focused on the synthesis of cobalt ferrite in the superparamagnetic region (where no significant enhancement of coercivity is possible) $)^{3,8,10-12}$ or polycrystalline multidomain particles, ${ }^{13,14}$ in which case coercivity is expected to decrease. To our understanding, there is still a lack of a systematic attempt to restrict the growth of single nanocrystals within the magnetic single domain region where enhancement of coercivity could be achieved. Accordingly,

a)Electronic mail: ycm2635@uprm.edu. we present a modified coprecipitation synthesis technique that controls the growth of cobalt ferrite single nanocrystals to achieve high room-temperature (RT) coercivity and moderate magnetization at the nanoscale. This size-controlled synthesis approach became possible by controlling the oversaturation conditions during ferrite formation in aqueous solutions. Optimum oversaturation was achieved by a suitable selection of synthesis conditions and precise monitoring of the feeding flow rate of reactant solutions under intensive heating conditions.

\section{EXPERIMENTAL}

\section{A. Materials}

All reagents were of analytical grade and were used without further purification. Required weights of $\mathrm{CoCl}_{2} \cdot 6 \mathrm{H}_{2} \mathrm{O}$ and $\mathrm{FeCl}_{3} \cdot 6 \mathrm{H}_{2} \mathrm{O}$ salts were dissolved in distilled water to produce a solution with $\mathrm{Fe}$ :Co mole ratio of $2: 1$. An aqueous solution of $\mathrm{NaOH}$ was used as the precipitant agent.

\section{B. Synthesis of cobalt ferrite nanocrystals}

The ferrite nanocrystals were synthesized by conventional and modified coprecipitation method. In the conventional approach, an aqueous solution of $0.055 M \mathrm{Co}(\mathrm{II})$ and $0.11 M \mathrm{Fe}$ (III) was rapidly contacted with an excess of hydroxide $\left(\mathrm{OH}^{-}\right)$ions. The hydrolysis reaction in the presence of an excess of $\mathrm{OH}^{-}$ions leads to the formation of a paramagnetic $\mathrm{Fe}-\mathrm{Co}$ hydroxide, which undergoes dehydration and atomic rearrangement conducive to a ferrite structure. The reactant solution was intensively heated to acelerate the 


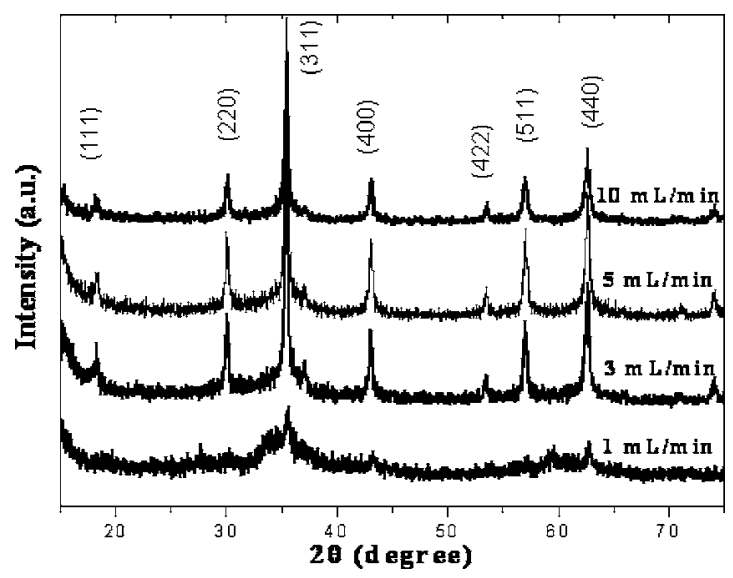

FIG. 1. XRD patterns for $\mathrm{CoFe}_{2} \mathrm{O}_{4}$ powders synthesized at different flow rates. The reaction time was $60 \mathrm{~min}$.

ferrite formation. Cobalt ferrite nanocrystals were washed out with distilled water, dried in air at $80^{\circ} \mathrm{C}$, and characterized. In order to enhance the magnetic properties of the crystal by promoting their growth, the control of the oversaturation conditions in the reaction solution was attempted. Accordingly, the conventional coprecipitation route was modified by controlling the flow rate of addition of the metal ions solution to the alkaline one under boiling conditions. ${ }^{5}$ For this purpose, a microperistaltic pump with precise control of flow rate was used.

\section{Nanocrystal characterization}

Dried powders were characterized using x-ray diffraction (XRD) and superconducting quantum interference device (SQUID) techniques. The average crystallite size of produced powders was estimated by using Scherrer's equation for the (440) peak. Transmission electron microscopy (TEM) and energy-dispersive $\mathrm{x}$-Ray spectroscopy (EDS) in a CM200 UTWIN TEM unit were used to study the morphology, structure, and composition of the nanocrystals.

\section{RESULTS AND DISCUSSION}

\section{A. XRD Analyses}

XRD analyses confirmed the formation of the ferrite structure for a reaction time as short as $5 \mathrm{~min}$. The concentration of $\mathrm{NaOH}$ was $0.48 M$ for all these experiments. Under noncontrolled flow-rate conditions, i.e., by following the conventional coprecipitation approach, the average crystallite size varied from 12 to $14 \mathrm{~nm}$ as the reaction time was increased from 5 to $180 \mathrm{~min}$, respectively. Although minor, the increase in average crystallite size for longer reaction times could be attributed to crystal growth, favored by Oswald-ripening process. ${ }^{15}$ The ferrite lattice parameter was estimated at $8.38 \pm 0.02 \AA$, which is in good agreement with the bulk value of $8.377 \AA .{ }^{16}$ Figure 1 shows the XRD patterns for the ferrites produced at different flow-rates of reactants addition and $60 \mathrm{~min}$ of reaction. As seen, the crystallinity of the ferrite products was very sensitive to the variation in flow rate. Broad and noisy XRD peaks were observed for the sample synthesized at $1 \mathrm{ml} / \mathrm{min}$. This peak

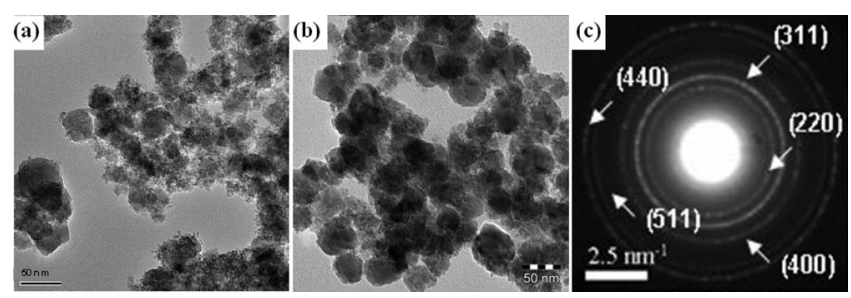

FIG. 2. TEM pictures for $\mathrm{CoFe}_{2} \mathrm{O}_{4}$ nanoparticles produced after 5 min (a) and $60 \mathrm{~min}$ (b) of reaction. The flow rate was $10 \mathrm{ml} / \mathrm{min}$. (c) TEM-ED pattern for $\mathrm{CoFe}_{2} \mathrm{O}_{4}$ nanoparticles synthesized after $10 \mathrm{~min}$ of reaction.

broadening suggests a poor development of the ferrite structure and was attributed to the extremely low saturation conditions established at such a low flow rate. In turn, welldefined and sharp peaks corresponding to the ferrite structure were observed for the solids synthesized at flow-rates above $1 \mathrm{ml} / \mathrm{min}$. The corresponding average crystallite size values varied from 31 to $24 \mathrm{~nm}$ for flow rates between 1 and $10 \mathrm{ml} / \mathrm{min}$, respectively. These sizes are almost 2.5 times larger than the average crystallite size obtained with no control of the flow rate. The establishment of suitable conditions for heterogeneous nucleation, where earlier nuclei should have acted as preexistent seeds, should have promoted crystal growth.

\section{B. TEM analyses}

TEM images of ferrite samples produced at $10 \mathrm{ml} / \mathrm{min}$ and 5 and 60 min of reaction are shown in Figs. 2(a) and 2(b), respectively. Particles were polydisperse; however, the promotion of crystal growth by prolonging the reaction time was evidenced. The size distribution was not determined because of the aggregation of the particles but particle sizes between 10 and $50 \mathrm{~nm}$ were observed. Figure 2(c) shows the electron diffraction (ED) patterns of nanocrystals produced at 10 min of reaction with no control of flow rate. All crystallographic planes in the ED pattern are in good agreement with those of $\mathrm{CoFe}_{2} \mathrm{O}_{4}$ evidencing the good crystallinity of the product. A standardless TEM-EDS analysis reported Co, $\mathrm{Fe}$, and $\mathrm{O}$ atomic percents of $13.04 \%, 29.57 \%$, and $57.39 \%$, respectively. These results are very similar to the theoretical ferrite composition $(\mathrm{Co}=14.28 \%, \mathrm{Fe}=28.57 \%, \mathrm{O}=57.14 \%)$.

\section{SQUID measurements}

Room-temperature $M-H$ measurements confirmed the influence of the synthesis conditions on the magnetic properties of ferrite nanocrystals. The coercivity was increased dramatically under controlled flow-rate conditions (Fig. 3). This parameter increased from $460 \mathrm{Oe}$, (no control on flow rate), up to 1597 Oe for $5 \mathrm{ml} / \mathrm{min}$ and $60 \mathrm{~min}$ of reaction. The corresponding maximum magnetization for this sample was $38 \mathrm{emu} / \mathrm{g}$. The magnetization went up to $57 \mathrm{emu} / \mathrm{g}$ for the solids produced with a flow rate of $10 \mathrm{ml} / \mathrm{min}$. The presence of superparamagnetic particles is suggested by the lack of saturation of the $M-H$ curve, even for an applied field as high as $5 \mathrm{~T}$. In turn, the drastic enhancement in coercivity can be attributed to the promotion of crystal growth within the single domain region, as suggested by XRD and TEM analyses. 


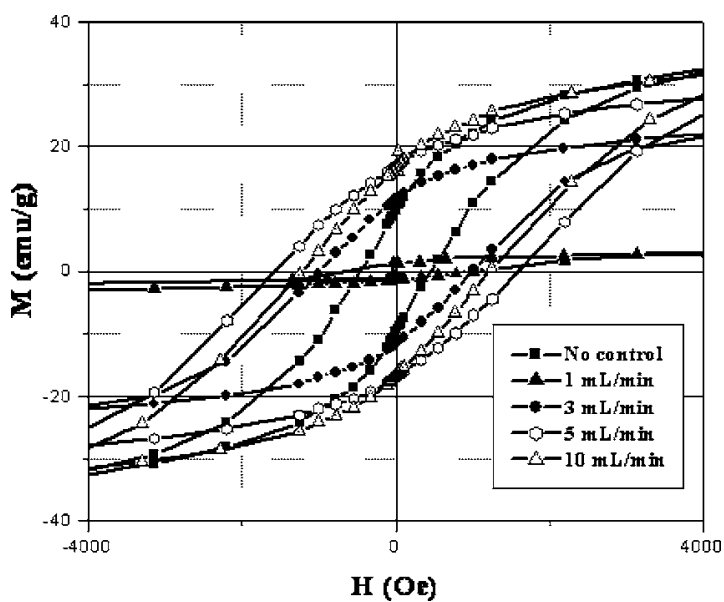

FIG. 3. $M-H$ loops at $300 \mathrm{~K}$ for $\mathrm{CoFe}_{2} \mathrm{O}_{4}$ synthesized at different flow rates. The reaction time was $60 \mathrm{~min}$.

\section{Factorial design}

A factorial statistical design permits the simultaneous examination of the effects of independent variables (synthesis parameters) and the interactions between them on a response (dependent variable). ${ }^{17,18}$ Accordingly, a $2^{3}$ factorial design - that considered three parameters and two levels for each one-was used to determine which synthesis parameters would exert more influence on the coercivity of ferrite nanocrystals. The levels of the synthesis parameters in the factorial design were defined based on the trends observed in the previous experiments. Accordingly, to investigate the effect of the reaction time, flow rate and $\mathrm{NaOH}$ concentration two levels for each one of these parameters $(5$ and $180 \mathrm{~min}$, 0.85 and $20 \mathrm{ml} / \mathrm{min}, 0.34 M$ and $0.54 M$, respectively) were considered. All these experiments were carried out under intensive heating conditions, where a burner was used instead of a hot plate. Table I summarizes the experimental conditions and the obtained coercivity values (dependent variable). In this table, the high and low levels of the synthesis parameters are represented by the $(+)$ and $(-)$ signs. For example, 180 and $5 \mathrm{~min}$ were the high and low levels, respectively, for the reaction time. These experiments were complemented with three replicates at the center of the design to estimate the experimental error. Coercivity values between 870 Oe (combination "ab:" $180 \mathrm{~min}$ of reaction, flow rate of $20 \mathrm{ml} / \mathrm{min}$, and $\mathrm{NaOH} 0.34 \mathrm{M}$ ) and $4626 \mathrm{Oe}$ (combination "a:" $180 \mathrm{~min}$ of reaction, flow rate of $0.85 \mathrm{~mL} / \mathrm{min}$, and $\mathrm{NaOH} 0.34 \mathrm{M}$ ), were achieved. The average crystallite size for the nanocrystals with the highest coercivity was estimated at $20 \mathrm{~nm}$. The coercivity of $4.6 \mathrm{kOe}$ is close to the theoretical prediction of $5.3 \mathrm{kOe}$ calculated for a system of noninteracting single domain cobalt ferrite particles with cubic anisotropy ${ }^{19}$ and the $5.4 \mathrm{kOe}$ considered for
TABLE I. Experimental conditions for the $2^{3}$ factorial design and the corresponding response (room-temperature coercivity)

\begin{tabular}{|c|c|c|c|c|c|}
\hline Combinations & $\begin{array}{l}\text { A B C } \\
\text { Design }\end{array}$ & $\begin{array}{c}\text { A: } \\
\text { Reaction } \\
\text { Time } \\
(\mathrm{min})\end{array}$ & $\begin{array}{c}\text { B: } \\
\text { Flow } \\
\text { Rate } \\
(\mathrm{mL} / \mathrm{min})\end{array}$ & $\begin{array}{c}\mathrm{C}: \\
\mathrm{NaOH} \\
(\mathrm{M})\end{array}$ & $\begin{array}{c}\mathrm{H}_{c} \\
\text { (Oe) }\end{array}$ \\
\hline 1 & --- & 5 & 0.85 & 0.34 & 4518 \\
\hline $\mathrm{a}$ & +-- & 180 & 0.85 & 0.34 & 4626 \\
\hline b & -+- & 5 & 20 & 0.34 & 871 \\
\hline$a b$ & ++- & 180 & 20 & 0.34 & 870 \\
\hline $\mathrm{c}$ & --+ & 5 & 0.85 & 0.54 & 2877 \\
\hline ac & +-+ & 180 & 0.85 & 0.54 & 3448 \\
\hline $\mathrm{bc}$ & -++ & 5 & 20 & 0.54 & 922 \\
\hline$a b c$ & +++ & 180 & 20 & 0.54 & 1007 \\
\hline
\end{tabular}

bulk, i.e., microcrystalline cobalt ferrite. The statistical anlysis of the obtained data suggested that both the flow rate of addition of reactants and $\mathrm{NaOH}$ concentration were the most significant parameters at a level of confidence of $99 \%$ and $95 \%$, respectively.

\section{ACKNOWLEDGMENTS}

This material was based upon work supported by the National Science Foundation under NSF-PREM Grant No. 0351449 at UPRM and NSF-PREM Grant No. 0304479 at UW.

${ }^{1}$ F. Zhang, S. Kantake, Y. Kitamoto, and M. Abe, IEEE Trans. Magn. 35, 2751 (1999)

${ }^{2}$ R. Arulmurugan, G. Vaidyanathan, S. Sendhilnathan, and B. Jeyadevan, J. Magn. Magn. Mater. 298, 83 (2006)

${ }^{3}$ N. Moumen, P. Bonville, and M. P. Pileni, J. Phys. Chem. 100, 14410 (1996).

${ }^{4}$ A. E. Berkowitz and W. Schuele, J. Appl. Phys. 30, S134 (1959).

${ }^{5}$ C. N. Chinnasamy, B. Jeyadevan, O. Perales-Perez, K. Shinoda, K. Tohji, and A. Kasuya, IEEE Trans. Magn. 38, 2640 (2002).

${ }^{6}$ O. Perales-Perez, H. Sasaki, A. Kasuya, B. Jeyadevan, K. Tohji, T. Hihara, and K. Sumiyama, J. Appl. Phys. 91, 6958 (2002).

${ }^{7}$ M. H. Khedr, A. A. Omar, and S. A. Andel-Moaty, Colloids Surf., A 281, 8 (2006).

${ }^{8}$ Q. Song and Z. J. Zhang, J. Am. Chem. Soc. 126, 6164 (2004).

${ }^{9} \mathrm{~S}$. Li, V. T. John, C. O'Connor, V. Harris, and E. Carpenter, J. Appl. Phys. 87, 6223 (2000)

${ }^{10}$ T. Hyeon, Y. Chung, J. Park, S. S. Lee, Y.-W. Kim, and B. H. Park, J. Phys. Chem. B 106, 6831 (2002).

${ }^{11}$ E. Tirosh, G. Shemer, and G. Markovich, Chem. Mater. 18, 465 (2006).

${ }^{12}$ J.-G. Lee, J. Y. Park, and C. S. Kim, J. Mater. Sci. 33, 3965 (1998).

${ }^{13}$ B. H. Liu and J. Ding, Appl. Phys. Lett. 88, 042506 (2006).

${ }^{14}$ M. P. Gonzalez-Sandoval, A. M. Beesley, M. Miki-Yoshida, L. FuentesCobas, and J. A. Matutes-Aquino, J. Alloys Compd. 369, 190 (2004).

${ }^{15}$ I. M. Lifshitz and V. V. Slyozov, J. Phys. Chem. Solids 19, 35 (1961).

${ }^{16}$ T. Meron, Y. Rosenberg, and Y. Lereah, J. Magn. Magn. Mater. 292, 11 (2005).

${ }^{17}$ C. Prado, J. Garrido, and J. F. Periago, J. Chromatogr., B: Anal. Technol. Biomed. Life Sci. 804, 255 (2004).

${ }^{18}$ W.-D. Yang and C.-S. Hsieh, J. Mater. Res. 14, 3410 (1999).

${ }^{19}$ E. W. Lee and J. E. Bishop, Proc. Phys. Soc. London 89, 661 (1966). 